\title{
Parameter Optimization with Input/Output Data via DE for Adaptive Control System with Neural Network
}

\author{
Taro Takagi \\ Department of Control Engineering, Natl. Inst. of Tech., Maizuru College, \\ 234 Shiroya, Maizuru, Kyoto 625-8511, Japan \\ Ikuro Mizumoto \\ Graduate School of Science and Technology, Kumamoto University, \\ 2-39-1 Kurokami Chuo-ku, Kumamoto 860-8555, Japan \\ E-mail:t.takagi@maizuru-ct.ac.jp,ikuro@kumamoto-u.ac.jp
}

\begin{abstract}
In this paper, adaptive control system with neural network $(\mathrm{NN})$ will be designed. At the beginning, parallel feedforward compensator (PFC) will be designed by using one-shot experimental data of controlled system via differential evolution (DE). From the obtained PFC and the ideal almost strictly positive real (ASPR) model, nominal model of controlled system can be obtained. Then, parameters of adjust law for NN will be optimized by using obtained nominal model via DE.
\end{abstract}

Keywords: Adaptive Control, ASPR, PFC, Neural network, Differential evolution.

\section{Introduction}

In spite of their simple structure, Almost strictly positive real (ASPR) based adaptive control systems have high robustness with respect to disturbances and uncertainties ${ }^{1}$, 2. However, the ASPR conditions which controlled system has to satisfy are very severe conditions for actual systems. To overcome this problem, introduction of parallel feedforward compensator (PFC) has been proposed $^{3}$. Several design methods of PFC which are based on mathematical model of controlled system such as relative degree have been proposed ${ }^{4}$. However, to obtain the mathematical model, iterative of identification experiments have to be done and it cost time and money. Therefore, a design method which is using system's input/output data has been proposed ${ }^{5,6}$. For ASPR system, an adaptive control system with feedforward input generated by neural network (NN) has been proposed ${ }^{7}$. This input is applied to avoid tracking error caused by PFC. In theory, PFC and NN parameters can be any value if the conditions are satisfied. However, those parameters will directly affect control performance. Therefore, it has to be appropriate value in actual control systems. Since there are no decision method for those parameters, designer has been decided it by trial and error of numerical simulations. Therefore, it is quite difficult to decide appropriate value.

In this paper, the parameter optimization method of PFC and NN by using input/output data via differential evolution (DE) will be proposed. DE is one of the evolutionary computation methods and it has a good convergence and uniqueness of solution ${ }^{8}$.

\section{Adaptive Control System with Adaptive NN Feedforward Input}

Consider the controlled system is described in equation state model as follows.

$$
\begin{aligned}
\dot{\boldsymbol{x}}(t) & =A \boldsymbol{x}(t)+\boldsymbol{b} u(t) \\
y(t) & =\boldsymbol{c}^{\top} \boldsymbol{x}(t)
\end{aligned}
$$


For this system, a PFC described as follows is introduced.

$$
\begin{aligned}
\dot{\boldsymbol{x}}_{f}(t) & =A_{f} \boldsymbol{x}_{f}(t)+\boldsymbol{b}_{f} u(t) \\
y_{f}(t) & =\boldsymbol{c}_{f}^{\top} \boldsymbol{x}_{f}(t)
\end{aligned}
$$

Then, supposed that the augmented system:

$$
\begin{aligned}
\dot{\boldsymbol{x}}_{a}(t) & =A_{a} \boldsymbol{x}_{a}(t)+\boldsymbol{b}_{a} u(t) \\
y_{a}(t) & =\boldsymbol{c}_{a}^{\top} \boldsymbol{x}_{a}(t)
\end{aligned}
$$

where

$$
\begin{gathered}
\boldsymbol{x}_{a}(t)=\left[\begin{array}{ll}
\boldsymbol{x}^{\top} & \boldsymbol{x}_{f}^{\top}
\end{array}\right]^{\top}, A_{a}=\operatorname{diag}\left[A A_{f}\right], \\
\boldsymbol{b}_{a}=\left[\begin{array}{ll}
\boldsymbol{b}^{\top} & \boldsymbol{b}_{f}^{\top}
\end{array}\right]^{\top}, \boldsymbol{c}_{a}^{\top}=\left[\begin{array}{ll}
\boldsymbol{c}^{\top} & \boldsymbol{c}_{f}^{\top}
\end{array}\right]
\end{gathered}
$$

is ASPR. Fig. 1. shows the block diagram of augmented system. Then, for this augmented system, design an adaptive output feedback control system with adaptive $\mathrm{NN}$ as follows.

$$
\begin{aligned}
u(t) & =u_{e}(t)+u_{n n}(t) \\
u_{e}(t) & =-k(t) \bar{e}_{a}(t) \\
u_{n n}(t) & =\hat{W}(t)^{\top} S(\omega) \\
\dot{k}(t) & =\gamma \bar{e}_{a}(t)^{2}-\sigma k(t) \\
\dot{\hat{W}}(t) & =-\Gamma S(\omega) \bar{e}_{a}(t)-\sigma_{n} \hat{W}(t)
\end{aligned}
$$

where

$$
\begin{gathered}
\bar{e}_{a}(t)=\bar{y}_{a}(t)-y_{r}(t) \\
\bar{y}_{a}(t)=y(t)+\bar{y}_{f}(t)
\end{gathered}
$$

and $\bar{y}_{f}(t)$ is the output of PFC obtained by

$$
\begin{aligned}
\dot{\bar{x}}_{f}(t) & =A_{f} \overline{\boldsymbol{x}}_{f}(t)+\boldsymbol{b}_{f} u_{e}(t) \\
\bar{y}_{f}(t) & =\boldsymbol{c}_{f}^{\top} \overline{\boldsymbol{x}}_{f}(t)
\end{aligned} .
$$

Also, $\hat{W}=\left[\hat{w}_{1}, \cdots, \hat{w}_{l}\right]^{\top} \in \boldsymbol{R}^{l}$ is the estimated value of ideal weight vector of $\mathrm{NN}$ and $l$ is the number of $\mathrm{NN}$ nodes. In addition, $S(\boldsymbol{\omega})=\left[s_{1}(\boldsymbol{\omega}), \cdots, s_{l}(\boldsymbol{\omega})\right]^{\top}$ is a radial basis function vector generally designed by the Gaussian functions such as

$$
s_{i}(\boldsymbol{\omega})=\exp \left[\frac{-\left(\boldsymbol{\omega}-\boldsymbol{\mu}_{i}\right)^{\top}\left(\boldsymbol{\omega}-\boldsymbol{\mu}_{i}\right)}{\eta_{i}^{2}}\right]
$$

where $\boldsymbol{\mu}_{i}$ is the center of receptive field and $\eta_{i}$ is the width of Gaussian function. Fig. 2. shows the block diagram of adaptive control system which is shown in this section.

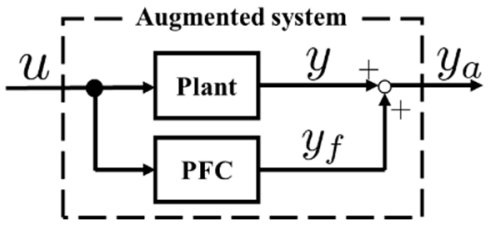

Fig. 1. Block diagram of augmented system.

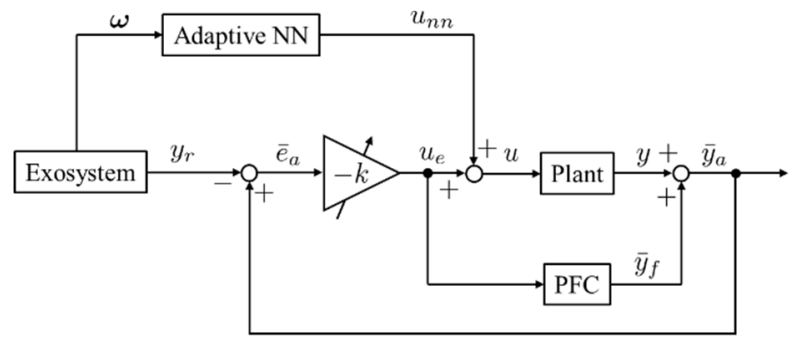

Fig. 2. Block diagram of adaptive control system with NN feedforward input.

Here, the problems are how to design the PFC and how to decide the parameters of adjusting laws.

\section{Design of PFC by Input/Output Data}

Define the ideal output of the ideal augmented system with the input data as follows.

$$
y_{a}^{*}(t)=G_{a}^{*}(s)[u(t)] .
$$

Then, the ideal output of PFC can be described by using the output of controlled system as follows.

$$
y_{f}^{*}(t)=y_{a}^{*}(t)-y(t) .
$$

Here, suppose that the ideal PFC given by the following:

$$
H^{*}(s)=\frac{N_{H}^{*}(s)}{D_{H}^{*}(s)}=\frac{b_{1}^{*} s^{n_{f-1}}+\cdots+b_{n_{f}}^{*}}{s^{n_{f}}+a_{1}^{*} s^{n_{f-1}}+\cdots+a_{n_{f}}^{*}}
$$

then the ideal output of PFC also can be given as

$$
y_{f}^{*}(t)=H^{*}(s)[u(t)] .
$$

By introducing a stable filter:

$$
F(s)=\frac{1}{s^{n_{f}}+f_{1} s^{n_{f-1}}+\cdots+f_{n_{f}}},
$$

Eq. (12) can be represented as

$$
y_{f}^{*}(t)=\frac{D_{H}^{*}(s)-F(s)}{F(s)} y_{f}^{*}(t)+\frac{N_{H}^{*}(s)}{F(s)}[u(t)]
$$

By defining the ideal parameters as

$$
\boldsymbol{\rho}^{*}=\left[\begin{array}{llllll}
z_{1} & \cdots & z_{n_{f}} & b_{1} & \cdots & b_{n_{f}}
\end{array}\right]^{\top} \quad\left(z_{i}=f_{i}-a_{i}\right)
$$

and the filtering signal as

$$
\boldsymbol{z}=\left[\frac{s^{n_{f}-1}}{F(s)} y_{f}^{*} \cdots \frac{1}{F(s)} y_{f}^{*} \frac{s^{n_{f}-1}}{F(s)} u \cdots \frac{1}{F(s)} u\right]^{\top}
$$

then the ideal output of PFC can be represented as

$$
y_{f}^{*}(t)=\boldsymbol{\rho}^{* \top} \boldsymbol{z}(t) .
$$

From eq. (9), for any parameters, the output of PFC can be given as

$$
y_{f}(t)=\boldsymbol{\rho}^{\top} \boldsymbol{z}(t) .
$$


Finally, consider minimizing the following evaluation function.

$$
J(\boldsymbol{\rho})=\int_{0}^{T}\left(y_{f}(t)-y_{f}^{*}(t)\right)^{2} d t .
$$

Although the order of PFC and parameters of filter affect to the output of PFC, there are no theorical method to decide these parameters. Therefore, it will be optimized by DE later. Remark that the ideal output of PFC is known but parameters of ideal PFC and filter are unknown.

\section{Differential Evolution}

In this section, the basic algorithm of DE which is applied in this study will be shown.

\section{(i) Creation of initial population}

Randomly create an initial $N$ sets of population as follows.

$$
\boldsymbol{X}_{i}^{(1)}=\left[X_{i, 1}^{(1)}, \cdots, X_{i, D}^{(1)}\right], i=1, \cdots, N .
$$

The superscript means a generation number of the vector, and $D$ is the order of decision variables.

\section{(ii) Extraction of base vector}

Extract the best vector from $n$th generation vector $\boldsymbol{X}_{1}^{(n)} \ldots \boldsymbol{X}_{N}^{(n)}$ which minimizing the evaluation function. This is, set the base vector as follows when the $l$ th target vector minimize the evaluation function.

$$
\boldsymbol{X}_{\text {base }}^{(n)}=\boldsymbol{X}_{\text {best }}^{(n)}=\boldsymbol{X}_{l}^{(n)} .
$$

\section{(iii) Mutation}

Generate a mutated vector from randomly chosen two vectors $\boldsymbol{X}_{r_{1, i}}^{(n)}, \boldsymbol{X}_{r_{2, i}}^{(n)}\left(r_{1, i} \neq r_{i, 2} \neq l\right)$ as follows.

$$
\boldsymbol{M}_{i}^{(n)}=\boldsymbol{X}_{\text {base }}^{(n)}+F\left(\boldsymbol{X}_{r_{1, i}}^{(n)}-\boldsymbol{X}_{r_{2, i}}^{(n)}\right) .
$$

Here, $F$ is a scaling factor for scaling the difference vector.

\section{(iv) Crossover}

Generate a trial vector from the target vector and the mutated vector as follows.

$$
\begin{gathered}
U_{i, j}^{(n)}= \begin{cases}M_{i, j}^{(n)} & \left(\operatorname{rand}_{i, j} \leq C \vee j=j_{\mathrm{r}}\right) \\
X_{i, j}^{(n)} & \left(\operatorname{rand}_{i, j}>C \wedge j \neq j_{\mathrm{r}}\right)\end{cases} \\
j=1,2, \cdots, D
\end{gathered}
$$

Here, $C$ is a crossover rate, $\operatorname{rand}_{i, j}$ is a random uniform numbers in the range $[0,1]$ and $j_{\mathrm{r}}$ is a randomly chosen integer number in the range $[1, D]$.

(v) Selection
Select the next generation as follows.

$$
\boldsymbol{X}_{i}^{(n+1)}=\left\{\begin{array}{l}
\boldsymbol{U}_{i}^{(n)}\left(f\left(\boldsymbol{U}_{i}^{(n)}\right) \leq f\left(\boldsymbol{X}_{i}^{(n)}\right)\right) \\
\boldsymbol{X}_{i}^{(n)}\left(f\left(\boldsymbol{U}_{i}^{(n)}\right)>f\left(\boldsymbol{X}_{i}^{(n)}\right)\right) .
\end{array}\right.
$$

The shown DE algorithm is so-called DE/best/1/bin strategy9.

\section{Control System Design via Differential Evolution}

Here, concrete designing method of control system via DE will be shown.

\section{(i) PFC design}

(a) Obtain the input/output data set of controlled system.

(b) Creation of initial population Randomly create an initial $\mathrm{N}$ sets of population as follows

$$
\boldsymbol{X}_{i}^{(1)}=\left[X_{i, 1}^{(1)}, \cdots, X_{i, 2 n_{f}}^{(1)}\right], i=1, \cdots, N
$$

(c) Extraction of base vector

Extract the best vector from $n$th generation vector as Eq. (21) which minimizing the evaluation function Eq. (19) by using obtained input/output data.

(d) Mutation

Generate a mutated vector from randomly chosen two vectors as Eq. (22).

(e) Crossover

Generate a trial vector from the target vector and the mutated vector as Eq. (23).

(f) Selection

Select the next generation as Eq. (24).

\section{(ii) Adaptive controller design}

(a) Obtain an approximation model of controlled system

From ASPR model $G_{a}^{*}(s)$ and obtained PFC $H(s)$ in above step as

$$
\tilde{G}_{p}(s)=G_{a}^{*}(s)-H(s)
$$

(b) Creation of initial population

Randomly create an initial $\mathrm{N}$ sets of population as follows

$$
\boldsymbol{X}_{i}^{(1)}=\left[\gamma_{i}, \sigma_{i}, \operatorname{diag}\left(\Gamma_{i}\right), \sigma_{n i}, \eta_{i}, \mu_{i}\right]
$$

(c) Extraction of base vector

Extract the best vector from $n$th generation vector as Eq. (21) which minimizing the evaluation function as follows. 


$$
J(\boldsymbol{\rho})=\int_{0}^{T}\left(y_{r}(t)-y(t)\right)^{2} d t
$$

(d) Mutation

Generate a mutated vector from randomly chosen two vectors as Eq. (22).

(e) Crossover

Generate a trial vector from the target vector and the mutated vector as Eq. (23).

(f) Selection

Select the next generation as Eq. (24).

\section{Numerical Simulation}

Assume that input/output data shown in Fig. 3 are obtained. From these data, by setting the parameters $F=$ 0.8 and $C=0.9$ with ASPR model

$$
G_{a}^{*}(s)=\frac{0.5}{s+1}
$$

third order PFC was obtained as

$$
H(s)=\frac{0.7692 s^{2}+6.229 s+2.292}{s^{3}+12.15 s^{2}+22.8 s+7.639}
$$

Then the adaptive controller parameters were optimized.

The initial value of design parameters were

$\gamma=50.020, \sigma=0.05, \Gamma=50.008, \sigma_{n}=0.05, \mu=0.7879, \eta=50.009$

Then the optimized parameters were

$\gamma=100, \sigma=0.052, \Gamma=32.082, \sigma_{n}=0.049, \mu=-31.658, \eta=100$

Fig.4 and Fig. 5 show the control result by initial adaptive controller parameters and optimized parameters respectively. It can be seen that the control performance became better after optimizing the parameters.

\section{Conclusions}

In this paper, the parameter optimization method which using input/output data via DE was proposed. The numerical simulation shows the usefulness of the method.

\section{References}

1. I. Mizumoto, S. Ohdaira and Z. Iwai, Output feedback strictly passivity of discrete-time nonlinear systems and adaptive control system design with a PFC, Automatica, 46(9) (2010) 1503-1509.

2. T. Takagi and I. Mizumoto, Adaptive PID control system with adaptive $\mathrm{NN}$ feedforward for discrete-time systems, IEEJ Transactions on Electronics, Information and Systems, 134(9) (2014) 1175-1181 (in Japanese)

3. H. Kaufman, I. Barkana and K. Sobel, Direct Adaptive Control Algorithms $2^{\text {nd }}$ Ed., (Springer-Verlag New York, 1997)
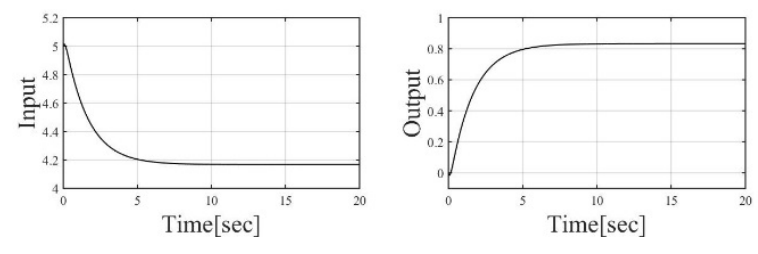

Fig. 3. Control input/output data.

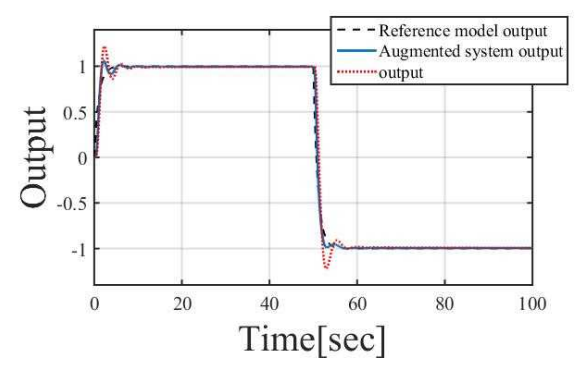

Fig. 4. Control result by initial parameters.

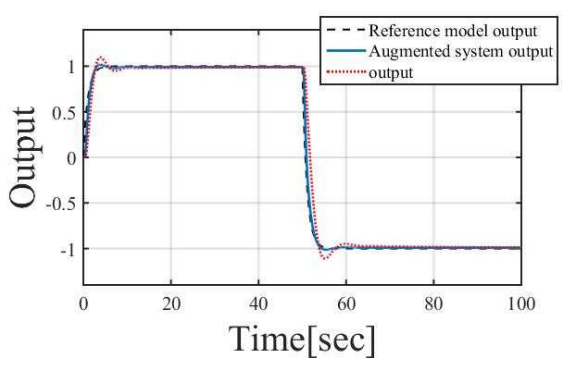

Fig. 5. Control result by optimized parameters.

4. Z. Iwai, I. Mizumoto and H. Ohtsuka, Simple Adaptive Control SAC, (Morikita Publishing, 2008)

5. I. Mizumoto and T. Takagi, PFC design via FRIT approach and adaptive PID control system design for discrete-time systems, In proc. of IFAC Conference on Advances in PID control, (2012) 64-69.

6. T. Takagi and I. Mizumoto, Adaptive output feedback control with adaptive PFC and its stability analysis, ICIC Express Letters, Part B: Applications, 6(12) (2015) 31893195.

7. I. Mizumoto, H. Tanaka and T. Tokimatsu, Adaptive output regulation of a class of discrete-time nonlinear systems based on output feedback and NN feedforward control, In proc. of 49th IEEE Conference on Decision and Control, (2010) 3631-3636.

8. M. Ito and M. Tanaka, A comparative study of particle swarm optimization, differential evolution, real-coded genetic algorithm on function optimization, Konan University Bulletin. Science and Technology, ed., 52(1) (2005) 125-135. (in Japanese)

9. G. C. Onwubolu, B. V. Babu, New Optimization Techniques in Engineering (Springer Science \& Business Media, 2004). 